\title{
Prácticas de liderazgo en una empresa manufacturera venezolana del sector cervecero
}

\author{
Zamora, Ánge ${ }^{*}$ \\ Poriet, Yenitza**
}

\section{Resumen}

El objetivo general de este artículo es determinar las prácticas de liderazgo en gerentes y supervisores en una empresa manufacturera. Los planteamientos abordados se refieren al uso de comportamientos que contribuyan a identificar y manejar el cambio, desarrollar y entusiasmar una visión, permitir que las personas asuman riesgos, fungir de modelos y celebración de los logros alcanzados. El diseño de investigación utilizado es de campo y de tipo descriptivo, contando con la participación de trece (13) sujetos, a quienes se le aplicó un cuestionario denominado Inventario de Prácticas de Liderazgo, derivando una alta confiabilidad, calculada mediante Alfa de Cronbach. Los resultados obtenidos indicaron que la práctica o conducta de liderazgo usada con mayor frecuencia por los líderes objeto de estudio fue: Brindar Aliento y le siguen: Servir de Modelo, Habilitar a otros para Actuar, Desafiar el Proceso e Inspirar una Visión Compartida; las cuales favorecen a alcanzar resultados extraordinarios en sus organizaciones. Concluyendo que éstas prácticas arrojadas en los resultados, son la clave para el desarrollo de la competitividad a nivel gerencial y organizacional.

Palabras clave: liderazgo, líderes, prácticas del Liderazgo, empresa manufacturera.

\section{Leadership Practices at a Manufacturing Company in the Brewing Sector}

\section{Abstract}

The general objective of this article was to determine the leadership practices used by managers and supervisors in a manufacturing company. Proposals refer to the use of behaviors that contribute to identifying and managing change, to developing and inspiring a shared vision, allowing people to take risks, acting as models and recognizing contributions. Research for this study was of a de-

\section{Recibido: 20.10.10. Aceptado: 26.02.11}

* Profesor Agregado, Universidad de Carabobo. e-mail: azamora7@hotmail.com.

**Profesora Asociado, Universidad de Carabobo. e-mail: yporiet@gmail.com. 
scriptive, field design. Thirteen (13) people were asked to complete a questionnaire called the Leadership Practice Inventory, which showed a high degree of reliability calculated using Cronbach's Alpha. Results indicated that the leadership practices or behaviors used most frequently by the leaders in this study were: encouragement, followed by being a model, enabling others to act, challenging the process and inspiring a shared vision, which favor achieving extraordinary results in their organizations. In conclusion, the results of these practices showed that they are keys for developing competitiveness at the managerial and organizational level.

Key words: leadership, leaders, leadership practices, manufacturing company.

\section{Introducción}

El tema del liderazgo y de los líderes tienen una importancia relevante dentro de las organizaciones sean estas del sector de manufactura y/o servicios, concentrando su atención y esfuerzos por tratar de entender y aplicar su significado, y las cosas que hacen a fin de obtener resultados. Los mismos están orientados a descifrar que significado tiene y también que usos y contribuciones se pueden extraer de su comprensión.

En este orden de ideas, la presente investigación tiene como objetivo general determinar las Prácticas de Liderazgo en gerentes y supervisores en una empresa manufacturera venezolana del sector cervecero presentando también como objetivos específicos los siguientes: Analizar los fundamentos teóricos-prácticos planteados por los autores Kouzes y Posner (1997, 2001), en cuanto a las Prácticas de Liderazgo. E Identificar las conductas de liderazgo de los gerentes y supervisores de la empresa objeto de estudio.

En tal sentido, la presente investigación se ha centrado en una organización venezolana perteneciente al sector manufacturero. La población estudiada corresponde principalmente al área estratégica de Mercadeo y Ventas. Obviamente, para esta área y la empresa en general, la percepción del consumidor debe ser un componente clave en sus negocios, la cual implica no confundir los índices de satisfacción del cliente con el compromiso de marca y los índices de ventas con los pronósticos comerciales.

La organización objeto de estudio se encuentra en un mercado que ha desarrollado altos niveles de resultados extraordinarios; en tal sentido, es relevante determinar las prácticas de liderazgo ejercidas por sus gerentes y supervisores, ya que en éstos recae -por supuesto, junto a sus colaboradores- la gran responsabilidad de que la organización y sus productos brinden altos niveles de satisfacción a sus clientes.

Otro aspecto interesante, es que en diferentes partes del mundo como USA, Europa, Australia, Asia y México se han desarrollado estudios a través de la aplicación del Inventario de Prácticas de Liderazgo (IPL).

Este inventario fue desarrollado por Kouzes y Posner (1997, 2001), con el fin de evaluar las cinco prácticas de liderazgo: Desafiar el Proceso, Inspirar una Visión Compartida, Habilitar a Otros para Actuar, Servir de Modelo y Brindar aliento.

La versión del IPL contiene treinta declaraciones y los autores Kouzes y Posner (2001) han desarrollado dos formas el IPL, "USTED" que es contestado 
para evaluar la conducta de liderazgo de un individuo en particular y el "OBSERVADOR" que es contestado por cinco o seis personas familiarizadas con la conducta de liderazgo de ese individuo.

La usada en esta investigación, utiliza una escala de Likert (1932) con diez puntos, de manera de designar cuan a menudo el líder usa un determinado comportamiento. Esta escala va desde 1 hasta 10 , donde el 1 significa, casi nunca, y el 10 casi siempre. Los valores para cada práctica van a situarse entre 6 y 60 . Las investigaciones, que se han realizado al efecto, muestran consistentemente, que la frecuencia con que los líderes utilizan estos comportamientos -que describen el IPL-sean utilizados, les permitirán ser percibidos como líderes efectivos.

Entre los beneficios que pueden generarse de esta investigación, se encuentran constituir una muestra de las acciones de los líderes en el contexto venezolano y poder hacer comparaciones en el ámbito nacional e internacional, de tal manera que permita generar aportes en el ámbito organizacional. Las características metodológicas corresponden, a una investigación de carácter descriptiva con diseño de campo no experimental. La recolección de información se efectuó directamente de una realidad, aplicando sin manipulación o control de variable alguna; el Inventario de Prácticas de Liderazgo (IPL)-USTED. Para determinar el grado en que los ítems del mismo eran homogéneos y consistentes, se efectuó el cálculo de Alfa de Cronbach, arrojando un resultado de 0,87 de confiabilidad, la cual es considerada como alta ya que es mayor de 0,70.

El colectivo a investigar en este estudio está integrado por gerentes y super- visores. La población total de esta investigación está representada por trece (13) personas, quienes son ocupantes de cargos a nivel gerencial y de supervisión, específicamente los adscritos al área de Mercadeo y Ventas de la organización objeto de estudio.

\section{Algunas consideraciones sobre liderazgo}

Una de las propuestas de investigación acerca de lo que hacen los líderes, es la de los autores Kouzes y Posner (1997, 2001), quienes han desarrollado fundamentos y planteamientos sobre Prácticas de Liderazgo, así como el instrumento: el Inventario de Prácticas de Liderazgo (IPL) (1997, 2001). Este inventario ha sido aplicado con éxito en diferentes culturas y ambientes laborales de organizaciones privadas y del sector público.

La premisa en que se apoya este modelo de liderazgo y sus cinco prácticas, como lo son: Desafiar el Proceso, Inspirar una Visión Compartida, Habilitar a otros para Actuar, Servir de Modelo y Brindar Aliento; tiene una conexión entre estas conductas que despliegan los líderes y lo que se considera un liderazgo exitoso.

A nivel del ámbito organizacional, esta investigación podría derivar aportes que conduzcan al desarrollo de estrategias de negocios más competitivas, dirigidas a generar resultados con valor agregado. Es importante considerar lo que señala Alves (2000), en cuanto a la necesidad de romper esquemas del modelo tradicional autoritario del liderazgo en América Latina, donde al líder se le ha exigido 
ser un individuo omnipotente y omnisciente, para convertir el ejercicio del liderazgo en la integración de lo analítico y lo afectivo.

Al hacer referencia al tema de los líderes y del liderazgo, necesariamente se vinculan estas dos palabras. Las mismas siguen generando bastante confusión en las exigentes organizaciones de este nuevo milenio; ya que en ellas hay hombres y mujeres que deben liderar a sus miembros, factor fundamental para incrementar constantemente sus niveles de producción y productividad, aunado a la persistente necesidad de dar satisfacción a las necesidades de servicio de sus clientes.

En reiteradas ocasiones el término liderazgo se ha tomado bajo la visión jerárquica, asociada a la gerencia e imposición de la autoridad; sin embargo, diversos estudios y enfoques sobre éste aspecto plantean que autoridad y liderazgo son términos que están relacionados, pero existen distinciones entre ellos.

Al respecto, Gil'Adi (1992:30) señala: "Ambas utilizan los recursos del poder, que es un producto de la autorización formal, dado por el título, cargo, posición conferida por la empresa, a desempeñar por el gerente en cuestión. Y también utilizan influencia, que es un producto de la autorización informal, dado por los subalternos a la persona que la gerencia, a través del convencimiento, dirección, canalización de intereses y expectativas de la gente. Por lo tanto, el liderazgo puede verse como un set de actividades entre las que está la de movilizar a las personas a trabajar en sus problemas, y la autoridad es una actividad que viene a resta- blecer o mantener el equilibrio del sistema social".

El tema del liderazgo ha sido estudiado desde diferentes ópticas, pretendiendo dar respuestas a los cambios y facilitar la adaptación de las organizaciones y sus miembros, en este siglo XXI, cuya característica fundamental es la incertidumbre.

En relación a estos diversos enfoques sobre liderazgo. Gil'Adi (1992:29), señala:

Primero, los talentos naturales son importantes. Segundo, la idea situacional es fundamental para entender que el liderazgo depende del contexto. Tercero, ciertos individuos pueden ser más exitosos si su predisposición se ajusta a la situación, especialmente si pueden identificar sus debilidades y talentos. En algunas situaciones es necesaria la conducta "autocrática", mientras en otras se requieren procesos participativos. Cuarto, el enfoque transaccional es esencial en el entendimiento de la influencia ejercida por seguidores y líder, uno sobre otro, a veces simultáneamente.

De acuerdo a estos planteamientos se puede asumir que el liderazgo es una habilidad relacionada con influencia, derivada del talento personal, dependiendo de la situación y/o contexto a través de habilidades de transacción.

Sin embargo, estos enfoques presentan "vacíos" en cuanto a estudios precisos sobre ¿Qué hacen los líderes?, ¿Cuáles son sus funciones? En este sentido, Gil'Adi (1992:29) expresa: "Yo creo que el análisis de las herramientas y métodos de liderazgo requiere de un contexto para su aplicación y, por lo tanto, la 
educación de los gerentes obliga primero a preguntarnos qué funciones necesitamos que un liderazgo ejerza".

Esta temática ha despertado gran interés en diferentes estudiosos del tema de liderazgo y entre ellos se encuentran Kouzes y Posner (1997:73), quienes definen el liderazgo ejemplar:"como el arte de movilizar a otros para que deseen luchar en pos de aspiraciones comunes". Estos autores han orientado sus investigaciones hacia las Prácticas de Liderazgo; tópico que en este nuevo milenio aumenta la necesidad de dar respuestas y aportes significativos, ya que como lo plantean Wright et al. (2000), es necesario pasar del pensamiento a la acción, ganar credibilidad cumpliendo sus promesas y demostrando logros y resultados.

A los líderes del siglo XXI se les exige un rol decisivo en el éxito de sus organizaciones, aún cuando hay autores que opinan que no son necesarios entre ellos destacan Howell et al. (1990); Pfeffer, (1977); consideran que los lideres deben poseer una mentalidad amplia, estar conscientes del papel que juegan en la organización, saber hacia dónde dirigir las estrategias que deben desarrollar y sobre todo sustentarlas en la credibilidad que proyecten en sus equipos.

Según Brooking (1997), Davenport (1999), Friedman, (et alt) (2000). En este nuevo entorno, no solo tienen valor los activos tangibles: propiedades, instalaciones y equipos; sino que en estos tiempos también demandan gran valor los activos intangibles: la gente, sus conocimientos y las relaciones armoniosas entre ellas.
La sociedad del nuevo milenio plantea como reto la transformación del liderazgo actual, en tal sentido es relevante investigar ¿Cuáles son las prácticas de liderazgo que contribuyen al éxito organizacional? ¿Cuáles acciones de los líderes permiten afrontar adecuadamente la globalización, la tecnología, la información y la gerencia del conocimiento?

Entre las premisas más relevantes para realizar esta investigación, se utilizo el Inventario de Prácticas de liderazgo (IPL), cuyos resultados generados al aplicar este instrumento, van a estar referidos a las acciones de estos líderes. Mientras que otros estudios, sin restarles importancia -ya que han efectuado aportes interesantes- lo que tratan es sobre los estilos de liderazgo (Poriet y Zamora: 1996).

\section{Enfoques sobre prácticas que deben desempeñar los líderes}

Para enfrentar los desafíos y retos del entorno organizacional, se requiere de la acción común de todos sus miembros, y esto a su vez implica la labor de alguien que abra el camino y oriente el esfuerzo de todos. En este sentido, hacen acto de presencia, individuos quienes a través de sus comportamientos movilizan a otros para la solución de problemas, facilitando su progreso para afrontar y adaptarse a las transformaciones de las organizaciones.

En este orden de ideas, se hace referencia al ejercicio del liderazgo; a las prácticas que desempeñan los líderes. Con relación a este aspecto, diferentes autores han planteado algunos compor- 
tamientos de liderazgo; entre ellos se encuentran:

Los autores Kets De Vries y Florent-Treacy (1999), consideran que los líderes de mayor éxito deben cumplir cuatro tareas principales: presentar estrategias globales, diseño de estructuras adecuadas, infundir valores, movilizar y motivar a una visión de futuro.

Kotter (1999) experto en temas de liderazgo, afirma que para liderar un cambio constructivo en una organización, se debe empezar por fijar una orientación en contraste como planificar y presupuestar; coordinar a las personas en contraste con organizar y dotar de personal; motivar a las personas en vez de controlar y resolver problemas, y crear una cultura de liderazgo.

Otro autor que presenta una propuesta bien iconoclasta acerca del papel de los líderes es Heifetz (1997). Este autor utiliza el término "movilizar", quien sostiene la idea de que la tarea más importante de los líderes en vista de los desafíos de las organizaciones por los cambios de ámbito mundial en las sociedades, mercados y tecnología, es la de movilizar a todos los trabajadores de las organizaciones a enfrentar y a trabajar en la "adaptación". Es decir, los trabajadores deben hacerse responsables, y no evitar, en trabajar en sus problemas.

También Heifetz y Laurie (1997:182): "ofrecen sus seis principios para liderar una labor de adaptación: Asomarse al Balcón, Identificar el Desafío de la Adaptación, Controlar la Angustia, Mantener una Atención Disciplinaria, Devolver el Trabajo a los Empleados y Proteger las Voces del Liderazgo que Provienen de Abajo".
Al estudiar lo planteado por estos autores, se pueden extraer los elementos comunes que presentan sus planteamientos, lo cual permite identificar que el papel de los líderes fundamentalmente consiste en movilizar a la gente; esto implica estimularlos para que se motiven, orientarlos e inspirarlos a afrontar sus problemas. En los enfoques estudiados sobre el papel de los líderes destacan áreas importantes como el aprendizaje, ser agentes de cambio y ayudar a enfrentar el "stress" en los seguidores.

Este liderazgo emergente no debe estar representado sólo en los individuos que ocupan cargos en las cúpulas de las organizaciones o en los mandos medios, incluso en supervisores de primera línea. Sino que también debe ser responsabilidad de todos sus integrantes, sin considerar la "posición" que ocupan en la estructura organizacional, cualquiera que sea el diseño de arquitectura que éstas utilicen.

Por tal razón la presente investigación se centra en el estudio de Prácticas de Liderazgo, según el modelo de Kouzes y Posner (1997), (2001). En virtud de que el mismo proporciona fundamentos teóricos-prácticos sobre los comportamientos del liderazgo; los cuales han sido recopilados durante más de diez (10) años por los autores mencionados, en los que han participado miles de líderes. Estos estudios han generado planteamientos y resultados bien interesantes, con énfasis en las conductas de los líderes que han participado en sus investigaciones. Ello le da un carácter práctico al estudio, generando así un modelo sobre lo que hacen los líderes; contribuyendo a comprender el proceso del liderazgo, y el 
desarrollo de las capacidades de las personas para liderar.

\section{Prácticas fundamentales del liderazgo ejemplar}

Las cinco prácticas fundamentales del liderazgo ejemplar identificadas en las investigaciones de Kouzes y Posner (1997), son: desafiar el proceso, inspirar una visión compartida, habilitar a otros para actuar, servir de modelo y brindar aliento; representan acciones dirigidas por los lideres a través de las cuales éstos logran realizar cosas extraordinarias, es decir, obtienen logros fuera de lo común.

De acuerdo a los estudios y experiencias adquiridas en las investigaciones efectuadas por estos autores, estas cinco prácticas no son exclusividad de unos pocos; las mismas están a disposición de cualquier persona que acepte el desafío del liderazgo, en diferentes organizaciones o situaciones. A continuación se describe casa uno de ellos.

\section{Desafiar al proceso}

Kouzes y Posner (1997): El éxito al desafiar y romper tradiciones, tener la iniciativa de cambiar las formas aceptadas de hacer las cosas, es muchas veces atribuidas a la suerte, pero es sólo a través de un proceder sostenido y constante, que el éxito es posible de ser alcanzado. Como decía Voltaire: "Suerte es lo que sucede cuando la preparación y la oportunidad se encuentran". Se ha llegado a una conclusión bien interesante entre los autores, que en el proceso de crear e innovar nuevas cosas, ello no implica nece- sariamente, que los líderes realmente creen nuevos productos, servicios o procesos; la mayoría de las veces, éstos vienen de agentes externos a la organización, como clientes y proveedores o por sucesos fortuitos a ellas. Lo que los líderes hacen, es brindar el apoyo, para que éstas nuevas propuestas se puedan poner en práctica y experimentan con ellas. La práctica de desafiar el proceso incluye las siguientes estrategias o compromisos:

- Salir a la búsqueda de oportunidades que presenten el desafío de cambiar, crecer innovar y mejorar.

- Experimentar, correr riesgos y aprender de los errores que se producen.

Cuando se habla de liderazgo y del papel que juegan los líderes en buscar nuevas formas de hacer las cosas y de mejorarlas; lo que éstos hacen, es tener la iniciativa que se requiere para enfrentar el desafío de cambiar el status quo. Es sólo a través de este tipo de comportamiento, que una persona, independientemente de su posición, pueda llegar a convertirse en líder. Los líderes que desafían el status quo, muchas veces tienen que romper paradigmas y hacer rupturas con el pasado y con la forma tradicional de hacer las cosas.

\section{Inspirar una visión compartida}

En el fondo inspirar una visión compartida, tiene que ver con lograr que otros se sientan identificados y comprometidos en una causa común, la visión que presente el líder debe ser lo suficientemente estimulante, comunicada con optimismo, entusiasmo y amor; de forma 
tal, que haga sentir a las personas a quien se comunica este mensaje, como triunfadores. Esta visión debe resultar creíble y posible, y el éxito de los líderes en lograr este compromiso con su gente, requiere, de que estos se perciban como personas en quienes se puede depositar su confianza.

Tal cual lo exponen Kouzes y Posner (2001:25): "Es la posición que le otorga a los líderes su autoridad, pero son sus comportamientos los que le hacen ganar el respeto. $Y$ esta consistencia entre las palabras y las acciones es lo que constituye la credibilidad de un líder".

En el modelo que presentan Kouzes y Posner (1997), (2001), en cuanto a la práctica de inspirar una visión compartida, ésta consiste en las siguientes estrategias:

- Imaginar un futuro edificante y ennoblecedor y

- Reunir a otros en torno a una visión común apelando a sus valores, intereses, sueños y esperanzas.

Los autores de esta propuesta de liderazgo, al hacer referencia a la capacidad que tienen los líderes de imaginar un ideal, prefieren utilizar el término visión.

\section{Habilitar a otros para actuar}

Kouzes y Posner (1997). Es una práctica de los líderes que implica trabajo en equipo, confianza en éste y la cesión del poder. Para los colaboradores o poderdantes de los líderes ésta es una capacidad fundamental del líder y la más significativa de las cinco prácticas, ya que el liderazgo es una relación basada en la confianza; ésta impulsa a la gente a co- rrer riesgos y éstos a los cambios para generar dinamismo y desarrollo de las organizaciones, Sin confianza ni cesión de poder del líder a su equipo, los cambios son prácticamente nulos y por tanto, las organizaciones estáticas y en dirección al deterioro y decadencia.

De acuerdo a Kouzes y Posner (1997), habilitar a otros para actuar es una práctica de liderazgo que comprende las siguientes estrategias o compromisos:

- Fomentar la colaboración: promover metas cooperativas y confianza mutua.

- Fortalecer a los demás: compartir el poder y la información.

\section{Servir de modelo}

Esta práctica de liderazgo conlleva a que los líderes a través de su ejemplo aprovechen cada oportunidad para demostrar que están profundamente comprometidos; además, que hagan tangibles las visiones y los valores.

La credibilidad del líder implica que las personas escuchen sus palabras y luego observan los actos; obviamente, evalúan la coherencia entre ambos, y si ésta se encuentra, el líder es considerado creíble, en caso contrario no es considerado una persona seria ni sincera. Al respecto Kouzes y Posner (1997:327) señalan que: "Por lo tanto, los actos son la evidencia del compromiso del líder. Esta observación conduce a un precepto para servir de modelo: Haga lo que dice que hará".

Los elementos esenciales de este precepto son: decir y hacer. 
Decir se refiere a los valores que deben poseer los líderes, éstos deben tenerlos claros y saber qué defienden. Hacer significa poner en práctica lo que dicen, deben actuar en base a sus creencias. Sin embargo, esto no es suficiente; para dar el ejemplo, buscando ganar y reforzar la credibilidad en el liderazgo, es necesario que el líder fundamente sus actos en un conjunto colectivo de objetivos y aspiraciones. Esto deriva a: "hacer lo que decimos que haremos" (Kouzes y Posner, 1997).

\section{Brindar aliento}

La posibilidad de lograr resultados que realmente aporten valor agregado en las tareas y procesos ejecutados por las personas, requiere de gran esfuerzo. Con el fin de mantener las esperanzas y la determinación de hacer contribuciones que valgan la pena, los líderes deben dar reconocimiento y premiar a las personas, tanto a nivel individual como a nivel de los equipos de trabajo, por el logro del alcance de objetivos extraordinarios.

La forma tradicional que se ha venido utilizando para premiar a las personas por dichos logros, es a través de recompensas materiales. A lo largo de los años, se han diseñado formas de evaluación de desempeño y de otorgamiento de incentivos por desempeño, como una forma de reconocer la dedicación y el esfuerzo de las personas que participan en el alcance de estos objetivos.

La distribución de dichos incentivos se ha planificado, y se han otorgado en términos de los aportes realizados. Otra forma también de satisfacer esa necesi- dad de reconocimiento por los logros alcanzados, que se viene utilizando con bastante frecuencia; tiene que ver con premiar los logros alcanzados denominados como incentivos no materiales. Estos consisten en celebrar los triunfos por los objetivos alcanzados.

Existe una variedad de formas de premiar a los trabajadores, con lo que se ha venido a denominar, como incentivos no materiales. Entre otros, estrechar la mano, dar palmadas en la espalda, el otorgamiento de placas y diplomas; se han venido utilizando como alternativas a los incentivos en metálico.

La práctica de Brindar Aliento incluye las siguientes estrategias:

- Reconocer las contribuciones individuales al éxito de cualquier proyecto.

- Celebrar los logros del equipo en forma regular.

La tendencia en general sin descuidar la motivación extrínseca y sus consecuencias en estimular el desempeño de los trabajadores; está en la de usar estrategias que promuevan la motivación intrínseca.

\section{Resultados del inventario de prácticas de liderazgo en una empresa manufacturera venezolana}

Haciendo uso del análisis documental, del contenido del material impreso y electrónico consultado, así como de la aplicación del instrumento, se efectuó el análisis de los fundamentos teóricosprácticos planteados por los autores Kouzes y Posner $(1997,2001)$, en cuanto a las Prácticas de Liderazgo, lo cual corres- 
ponde al primer objetivo de esta investigación.

Los resultados de las investigaciones desarrolladas por Kouzes y Posner (1997), (2001) han precisado que las Prácticas Fundamentales del Liderazgo Ejemplar son: Desafiar el Proceso, Inspirar una Visión Compartida, Habilitar a Otros para Actuar, Servir de Modelo y Brindar Aliento. (Estas tres últimas prácticas también se denominan permitir actuar a otros, modelar el camino y animar el corazón, respectivamente).

La práctica de los líderes Desafiar el Proceso se refiere a que éstos rompen tradiciones; para ello desarrollan iniciativa y asumen los riesgos necesarios que le permitan manejar el cambio que requieren las organizaciones y sus integrantes.

Inspirar una visión compartida es una práctica de liderazgo que consiste en visualizar el logro de cosas extraordinarias en situaciones comunes de la vida.

Los líderes al habilitar a otros para actuar, están otorgando y delegando su propio poder, están compartiendo información, proporcionando capacidad de decisión; con lo cual hacen que todos se sientan capaces y poderosos.

En cuanto a que los líderes sinen de modelo, ésta práctica enaltece la importancia de que los líderes deben dar el ejemplo para que otros los sigan, deben ser congruentes sus valores, con sus palabras y hechos.

En relación a la práctica Brindar Aliento o animar el corazón, se refiere a que los líderes deben reconocer y celebrar los triunfos en objetivos alcanzados. Esto implica los siguientes compromisos: reconocer las contribuciones indivi- duales al éxito de cualquier proyecto y celebrar los logros del equipo en forma regular.

Cada una de estas prácticas, para ser ejercidas por los líderes requiere del desarrollo de estrategias o compromisos; éstos se ha señalado en el contenido de la presente investigación. Un aspecto muy importante que se debe destacar es que tanto las prácticas de liderazgo como sus respectivos compromisos, son estrategias que pueden ser ejercidas por hombres y mujeres comunes y corrientes, con un día a día que implica logro de resultados; pero que marcan la diferencia al generar resultados extraordinarios o fuera de lo común, haciendo uso de estas prácticas.

Para aprender y desarrollar las mismas, los líderes ameritan de herramientas e ideas que contribuyan a desarrollar su capacidad para liderar, y sobre todo de esfuerzo, corazón y amor para marcar diferencia; esto es una premisa del modelo planteado por Kouzes y Posner $(1997,2001)$.

Con respecto al segundo objetivo específico de esta investigación: Identificar conductas de liderazgo de los gerentes y supervisores de una empresa venezolana, para el desarrollo del mismo se aplicó el Inventario de Prácticas de Liderazgo (IPL-“USTED”). Estos reportes o informes individuales se consolidan en la tabla 1.

En este estudio, para el análisis y la interpretación de la información obtenida del Inventario de Prácticas de Liderazgo (IPL-“USTED”), se consideraron los resultados de la tabla 2: Promedios, desviaciones estándar e índices de fiabilidad para el Inventario de Prácticas del Líder; 
la tabla 1: perfil del grupo participante; tabla 3: Perfiles individuales de calificaciones acumulativas.

En cuanto a las prácticas o conductas del liderazgo utilizadas por el colectivo investigado, se encontró lo siguiente:

Comparando los resultados de la tabla 1: con los valores presentados en la tabla 3, se detecta que en ambas resulta como práctica con una frecuencia de uso más baja por parte de los líderes: Inspirar una visión compartida. Con respecto a las otras cuatro (4) prácticas de liderazgo, los resultados de este estudio (tabla 1) en relación a los de la tabla 2, difieren en cuanto a la frecuencia de uso de esas prácticas.

En el colectivo investigado en este estudio se destaca como práctica de mayor uso, Brindar aliento, mientras que en la información de Kouzes y Posner (1997) aparece con mayor frecuencia la práctica Habilitar a otros para actuar (ver tabla 1 y tabla 2 , respectivamente). En la tabla 3 , al

\section{Tabla 1}

Inventario de Prácticas de Liderazgo. Perfil del grupo participante

\begin{tabular}{lcc} 
DESAFIAR EL PROCESO & MED & STD DEV \\
Autoevaluación & 42,2 & 5,6 \\
INSPIRAR UNA VISIÓN COMPARTIDA & MED & STD DEV \\
Autoevaluación & 39,8 & 6,1 \\
PERMITIR ACTUAR A OTROS & MED & STD DEV \\
Autoevaluación & 46,9 & 4,5 \\
MODELAR EL CAMINO & MED & STD DEV \\
Autoevaluación & 47,2 & 6,4 \\
ANIMAR EL CORAZÓN & MED & STD DEV \\
Autoevaluación & 49,7 & 4,7 \\
Número de evaluaciones de líderes & 13 & \\
\hline
\end{tabular}

MED: Media. STD DEV: Desviación Estándar.

Fuente: Autores (2003).

\section{Tabla 2}

\section{Promedios, desviaciones estándar e índices de fiabilidad para el Inventario de prácticas del líder}

\begin{tabular}{lccccc}
\hline \multicolumn{1}{c}{ Práctica de Liderazgo } & $\begin{array}{r}\text { Promedio Desviación } \\
\text { estándar }\end{array}$ & $\begin{array}{c}\text { IPL } \\
(\mathrm{N}=43,8999)\end{array}$ & $\begin{array}{c}\text { IPL-Propio } \\
(\mathrm{N}=6,651)\end{array}$ & $\begin{array}{c}\text { IPL-Observador } \\
(\mathrm{N}=37,248)\end{array}$ \\
\hline Desafiar el Proceso & 22,38 & 4,17 & 0,81 & 0,71 & 0,82 \\
Inspirar una visión compartida & 20,48 & 4,90 & 0,87 & 0,81 & 0,88 \\
Habilitar a otros para actuar & 23,89 & 4,37 & 0,85 & 0,75 & 0,86 \\
Servir de modelo & 22,18 & 4,16 & 0,81 & 0,72 & 0,82 \\
Brindar aliento & 21,89 & 5,22 & 0,91 & 0,85 & 0,92 \\
\hline
\end{tabular}

Fuente: Kouzes, J. y Posner, B., El Desafío del Liderazgo, 1997, Cuadro A1, (p. 516). 


\section{Tabla 3}

IPL-USTED. Perfiles individuales de calificaciones acumulativas

\begin{tabular}{cccccc}
\hline Sujetos & $\begin{array}{c}\text { Desafiar el } \\
\text { proceso }\end{array}$ & $\begin{array}{c}\text { Inspirar una visión } \\
\text { compartida }\end{array}$ & $\begin{array}{c}\text { Habilitar a otros para } \\
\text { actuar }\end{array}$ & $\begin{array}{c}\text { Servir de } \\
\text { modelo }\end{array}$ & $\begin{array}{c}\text { Brindar } \\
\text { aliento }\end{array}$ \\
\hline 1 & 46 & 44 & 49 & 48 & 46 \\
2 & 47 & 47 & 49 & 54 & 59 \\
3 & 40 & 34 & 42 & 52 & 54 \\
4 & 36 & 40 & 46 & 46 & 45 \\
5 & 50 & 44 & 48 & 40 & 49 \\
6 & 39 & 34 & 45 & 43 & 47 \\
7 & 34 & 48 & 46 & 46 & 52 \\
8 & 32 & 27 & 35 & 30 & 40 \\
9 & 44 & 36 & 54 & 51 & 51 \\
10 & 46 & 33 & 51 & 52 \\
11 & 39 & 45 & 48 & 54 \\
12 & 49 & 42 & 47 & 48 \\
13 & 46 & 43 & 50 & 50 & 53 \\
\hline
\end{tabular}

Fuente: Autores (2003).

Frecuencia de uso más baja __ Frecuencia de uso más alta

analizar EI IPL-USTED: Perfiles individuales de las calificaciones acumulativas de los trece (13) encuestados, se pudo evidenciar lo siguiente:

En el $69,23 \%$ de los encuestados, es decir, en nueve (9) de ellos, se identificó la práctica "inspirar una visión compartida", como la práctica con frecuencia de uso más baja.

En la tabla 1 se observa que los trece (13) sujetos que conforman el colectivo investigado, de acuerdo a la media y desviaciones estándar de cada una de las prácticas; demostraron una mayor inclinación en el uso de la práctica de liderazgo "Animar el Corazón" (también denominada "Brindar Aliento"), seguida de "Modelar el Camino" o "Servir de ModeIo", "Permitir actuar a Otros" o "Habilitar a otros para Actuar" y, "Desafiar el Proceso", siendo considerada la práctica: "Inspirar una Visión Compartida" como la de menos uso frecuente en el colectivo estudiado.

Una de las preguntas más usuales que surge cuando se utiliza un instrumento de esta naturaleza, como el Inventario de Prácticas de Liderazgo (IPL) Kouzes y Posner, (1997), (2001), tiene que ver con determinar que diferencia se observa realmente en una persona, cuando utiliza una práctica de liderazgo en particular y por la otra si ésta es importante.

Los autores Kouzes y Posner (1997, 2001) argumentan en este sentido que al igual que otros investigadores que han utilizado el Inventario de Prácticas de Liderazgo (IPL), no existen res- 
puestas universales, acerca de cuál práctica es mejor que otra. Lo que ellos afirman Kouzes y Posner (2001:26) es que el uso "frecuente como una persona se comporte consistentemente, de la forma tal cual es descrita en el IPL, ésta será percibida por otras personas como un líder eficaz".

Cuando se hace un análisis con el fin de identificar conductas de liderazgo, surgen a menudo preguntas tales como las que proponen Kouzes y Posner (2001:37):

“¿En cuál práctica obtiene el puntaje más alto? ¿En el segundo? ¿El tercero? ¿El cuarto? ¿El quinto?"

Estos puntajes indican áreas en las que el líder considera como sus fortalezas, y otros en los cuales podría considerar como proceso de mejora, a través de un plan de desarrollo.

La revisión de la tabla 1 Inventario de Prácticas de Liderazgo: Perfil del grupo participante, permite señalar importantes hallazgos, en cuanto al uso de las prácticas de liderazgo utilizadas por estos gerentes y supervisores objeto de estudio. Se observa cuando se hace el análisis de los valores de los promedios y las desviaciones estándar, lo siguiente:

Que en el caso de los resultados de esta investigación, el orden en que se ubican las cinco prácticas de liderazgo utilizadas por los trece (13) encuestados, refleja un orden distinto a los estudios realizados por Kouzes y Posner (1997), según tabla 2.

Como se puede observar la práctica de liderazgo más utilizada es la de "habilitar a otros para actuar", seguida de "desafiar el proceso", "servir de modelo", "brindar aliento", siendo la práctica de "inspirar una visión compartida" como la menos utilizada.

Considerando los hallazgos de la investigación, el liderazgo como figura estratégica debe centrarse en enfrentar y superar los desafíos, lo que se traduce en una búsqueda constante de resultados excepcionales. En la organización objeto de estudio su sistema de "desdoblamiento de metas anuales", permite a cada uno de sus miembros, conocer los alcances de sus contribuciones. En este sentido, la organización crea a través de un clima basado en la "confianza" de sus integrantes, asumir riesgos calculados en función de que cada quien conoce su nivel de contribución, a través de un sistema de alcanzar resultados excepcionales, pero respetando las normas básicas de ética de hacer negocios. Es como si siguieran la premisa de que "el fin justifica los medios".

Esto no sería posible de ser una realidad para una organización, si sus líderes no fomentaran valores de "realizar" y "hacer realidad", logros cada vez más retadores y novedosos. Por ello los líderes deben crear ambientes flexibles de trabajo que permitan el surgimiento de la creatividad y la innovación, de manera tal que, esos "sueños" sirvan del estímulo necesario que requieren las organizaciones para que sus miembros se sientan motivados a ser, más competitivos y al mismo tiempo seguros y dispuestos a enfrentar los retos en la búsqueda de soluciones novedosas y con alto valor agregado.

\section{Conclusiones}

En relación al estudio de campo desarrollado en esta investigación, se destaca las lo siguiente. 
Los retos generados por los cambios de paradigmas en las organizaciones, se deben principalmente al hecho de que el entorno en que éstas se desenvuelven, han cambiado drásticamente. Para enfrentar estos retos las organizaciones requieren de líderes que transmitan credibilidad y compromiso en sus asociados. De tal forma que a la vez que promuevan el pensamiento creativo, también los influencien, para que se dediquen a trabajar en pos de alcanzar objetivos de excelencia. Ello es posible de lograr, cuando sus valores individuales estén alineados con las actividades que realizan. Este liderazgo será más efectivo cuando los líderes muestren un genuino interés por contribuir en la satisfacción de las necesidades de sus asociados.

De acuerdo a los resultados y análisis efectuados en este estudio se puede precisar que no hay una práctica o conducta de liderazgo mejor que otra, las cinco prácticas en conjunto contribuyen al desarrollo de un liderazgo exitoso; en virtud de que la determinación de las prácticas del liderazgo utilizadas o desarrolladas por los líderes, es el punto de partida que permitirá desarrollar las capacidades y habilidades requeridas para un liderazgo ejemplar y que puede ser ejecutado por hombres y mujeres, que ocupan o no cargos gerenciales y de supervisión; pero que dentro de su contexto deban desarrollar la capacidad de liderazgo de: movilizar a otros para solucionar problemas y lograr cosas extraordinarias.

\section{Referencias bibliográficas}

Alves, Carlos. (2000). El Nuevo Liderazgo: EI gran Talón de Aquiles para la Geren- cia del Conocimiento. Revista ANRI. Recursos Humanos: Prioridad del Nuevo Milenio, 7 (Año 2). p. 11-12.

Brooking, Anne (1997). El Capital Intelectual. El Principal Activo de las Empresas del Tercer Milenio. $1^{\circ}$ edición, Ediciones Paidós lberica, S.A., España.

Davenport, Thomas (1999). Capital Humano. Creando Ventajas Competitivas a través de las personas. Ediciones Gestión 2000, S.A., 2000, España.

Friedman, Brian et al. (2000). Atraer, Gestionar y Retener. El Capital Humano. Cumplir lo Prometido. Ediciones Paidós Ibérica, S.A., España.

Gil'Adi, Daniel (1992). El Líder es el gran Movilizador de los Recursos Administrativos, Revista Enlace Químico, No. 2, Caracas, Venezuela, Enero-Marzo, p. 27-32.

Heifetz, Ronald (1997). Liderazgo sin Respuestas Fáciles. Ediciones Paidós Ibérica, S.A., España.

Heifetz, Ronald y Laurie Donald (1997). EI Trabajo del Liderazgo. En Harvard Business Review (1999). Bilbao, Ediciones Deusto, S.A., p. 181-210.

Howell, J. et al. (1990). Substitutes for Leadership: effective alternatives to Ineffective Leadership. Organizational Dynamics, 19, Number 1. P. 21-38.

Kets de Vries, Manfred y Florent-Treacy, Elizabeth (1999). Los Nuevos Líderes Globales. Editorial Norma, S.A. Colombia

Kotter, John (1999). La Verdadera Labor de un Líder. Harvard Business Review. Ediciones Deusto, S.A. España.

Kouzes, James y Posner, Barry (1997). El Desafío del Liderazgo. Ediciones Granica, S.A., España

Kouzes, James y Posner, Barry (2001). Leadership Practices Inventory (LPI). Revised Second Edition Facilitator's guide. Jossey-Bass Pfeiffer, USA. 
Likert, Rensis (1932). A Technique for the measurement of attitudes. New York. Mc. Graw-Hill.

Pfeffer, Jeffrey (1977). The Ambiguity of Leadership. Academy of Management Review. January 1977, p. 104-111, USA.

Poriet, Yenitza y Zamora, Angel (1996). Reflexiones sobre las Teorías del Lideraz- go. Trabajo de Ascenso para optar a las categorías de Profesor agregado y asistente en la Escuela de Relaciones Industriales de la Universidad de Carabobo. (No publicado).

Wright, Patrick et al. (2000). Nuevos Desafíos de la Gerencia Estratégica de Recursos Humanos. Creatividad, agilidad y acción. Ediciones IESA, C.A., Venezuela. 